\title{
Eficácia da simulação na autoconfiança de estudantes de enfermagem para ressuscitação cardiopulmonar extra-hospitalar: um estudo quase experimental
}

\author{
Effectiveness of simulation on nursing students' self-confidence for intervention \\ in out-of-hospital cardiopulmonary resuscitation: a quasi-experimental study
}

\author{
Genesis Souza Barbosa (D) 1,2 $\varangle$, Caio Guilherme Silva Bias (D) 3, Lorene Soares Agostinho (D) 2, \\ Luciana Maria Capurro de Queiroz Oberg (D) ${ }^{2}$, Rafael Oliveira Pitta Lopes ${ }^{(\mathbb{D} 4}{ }^{4}$, Regina Marcia Cardoso de Sousa (D) 1 \\ ${ }^{1}$ Universidade de São Paulo, Escola de Enfermagem, Departamento de Enfermagem Médico-Cirúrgica. São Paulo, SP, Brasil. \\ 2 Universidade Federal do Rio de Janeiro, Campus Macaé Professor Aloísio Teixeira, Área de Enfermagem Médico-Cirúrgica. Macaé, RJ, Brasil. \\ ${ }^{3}$ Instituto Nacional de Cardiologia. Rio de Janeiro, RJ, Brasil. \\ ${ }^{4}$ Universidade Federal do Rio de Janeiro, Campus Macaé Professor Aloísio Teixeira, Área de Fundamentos do Cuidado de Enfermagem e \\ História da Enfermagem Brasileira. Macaé, RJ, Brasil.
}

Como citar este artigo (How to cite this article):

Barbosa GS, Bias CGS, Agostinho LS, Oberg LMCQ, Lopes ROP, Sousa RMC. Eficácia da simulação na autoconfiança de estudantes de enfermagem para ressuscitação cardiopulmonar extra-hospitalar: um estudo quase experimental (Effectiveness of simulation on nursing students ' self-confidence for intervention in out-of-hospital cardiopulmonary resuscitation: a quasi-experimental study). Sci Med. 2019;29(1):e32694. https://doi.org/10.15448/1980-6108.2019.1.32694.

\section{RESUMO}

OBJETIVOS: Verificar a eficácia da simulação na autoconfiança de estudantes de enfermagem para ressuscitação cardiopulmonar extrahospitalar.

MÉTODOS: Estudo quase experimental, de grupo único, tipo antes e depois, realizado com estudantes de graduação em enfermagem. A amostra foi recrutada entre estudantes universitários que cursavam o segundo ou terceiro anos de graduação, e aceitaram participar da pesquisa. O protocolo de intervenção consistiu na participação individual em cenário clínico simulado de emergência. O cenário adotado consistiu numa ressuscitação cardiopulmonar em parada cardiorrespiratória extra-hospitalar, utilizando o manequim de baixa fidelidade Mini Anne Plus ${ }^{\circledR}$. Além das variáveis sociodemográficas, a autoconfiança dos estudantes para atuação em parada cardiorrespiratória foi avaliada pela Escala de Autoconfiança, antes e após a simulação. Foram aplicados os testes de homogeneidade marginal e de postos sinalizados de Wilcoxon e o nível de significância aceito foi de $5 \%$.

RESULTADOS: Trinta e dois estudantes de graduação em enfermagem na faixa etária entre 18 e 38 anos, participaram do estudo. Diferenças estatisticamente significantes $(\mathrm{p}<0,001)$ foram observadas nas respostas de todas as questões da Escala de Autoconfiança, quando comparadas antes e depois da simulação. Houve também aumento estatisticamente significativo $(\mathrm{p}<0,001)$ da pontuação nos domínios cardiológico, respiratório e neurológico após simulação.

CONCLUSÕES: A simulação se mostrou uma estratégia educativa eficaz no aumento da autoconfiança de estudantes de enfermagem para atuação em ressuscitação cardiopulmonar extra-hospitalar.

DESCRITORES: Enfermagem; Emergências; Simulação; Educação em Enfermagem; Ressuscitação Cardiopulmonar

\section{ABSTRACT}

AIMS: To verify the effectiveness of the simulation in the self-confidence of nursing students for extra-hospital cardiopulmonary resuscitation. METHODS: A quasi-experimental, before and after, single-group study, was performed with nursing undergraduate students. The sample was recruited among university students who were in the second or third year of graduation and accepted to participate in the research. The intervention protocol consisted of individual participation in a emergency simulated clinical scenario. The simulated scenario adopted consisted of cardiopulmonary resuscitation in extra-hospital cardiorespiratory arrest, using the Mini Anne Plus ${ }^{\circledR}$ low fidelity manikin. In addition to the sociodemographic variables, students' self-confidence for emergency action was analyzed, evaluated by the Self-Confidence Scale, before and after each simulation. Marginal and homogeneous Wilcoxon homogeneity tests were applied, and the accepted significance level was 5\%. RESULTS: Thirteen two undergraduate students in nursing between the ages of 18 and 38 participated in the study. Statistically significant differences $(p<0.001)$ were observed in the answers of all the questions of the Self-confidence Scale when compared before and after the simulation. There was also a statistically significant increase $(\mathrm{p}<0.001)$ in cardiological, respiratory and neurological scores after simulation CONCLUSIONS: The simulation proved to be an effective educational strategy in increasing the self-confidence of nursing students to perform extra-hospital cardiopulmonary resuscitation.

KEYWORDS: Nursing; Emergencies; Simulation; Education in Nursing; Cardiopulmonary Resuscitation. 


\section{INTRODUÇÃO}

As mudanças no modo de ensinar enfermagem na academia vêm reformulando a maneira com que experiências práticas são vivenciadas pelos estudantes [1]. Atualmente, os professores são incentivados a elaborar planos educacionais com objetivos claros voltados para melhorar o pensamento crítico e a autoconfiança de seus alunos [2]. Neste contexto, a autoconfiança é reconhecida como a capacidade que o indivíduo possui para, em um determinado ambiente, crer no sucesso de suas ações, por meio de suas próprias competências e habilidades psicomotoras, atitudinais e cognitivas [3].

A formação de enfermeiros implica em um processo que ultrapassa o acúmulo do saber de forma isolada, e trabalha para o desenvolvimento de um profissional que esteja constantemente usufruindo e relacionando teoria e prática. A associação dessas duas vertentes que compõem o processo de formação do enfermeiro permite que ocorra o desenvolvimento do pensamento crítico reflexivo, essencial para a atuação clínica $[4,5]$. É importante o desenvolvimento desse pensamento ainda na graduação para que os estudantes tenham melhor atuação em seu campo de prática e maior segurança ao lidar com seus pacientes [4].

Por vezes, sentimentos negativos dos estudantes com relação à prática clínica podem ser gerados por erros na correlação adequada da teoria e prática e pelo desenvolvimento crítico reflexivo insatisfatório. Insegurança, frustração, culpa, impotência, tristeza, desvalorização, opressão, dificuldade, perplexidade, nervosismo, constrangimento, vergonha, inutilidade, desmotivação e revolta são alguns dos sentimentos que foram relatados por estudantes no primeiro contato com a prática de ensino/estágio clínico [6]. Por outro lado, estudantes de enfermagem que foram expostos a um programa de prática em laboratório de simulação foram altamente propensos a ser competentes e confiantes em executar essas habilidades no ambiente de atendimento ao paciente [7].

Sobre esse aspecto, a simulação assume papel facilitador de processos de ensino-aprendizagem mais participativos, atendendo as singularidades de aprender dos estudantes e mantendo a condição dialógica que respeita sua autonomia no processo de aquisição do conhecimento [8].

A simulação é considerada um processo dinâmico que se baseia na criação de uma situação hipotética que incorpora a representação fiel da realidade, facilita o envolvimento do estudante e integra as complexidades envolvidas no processo de aprendizagem, prático e teórico, com oportunidade de repetição, feedback, avaliação e reflexão [9].

Ao longo da graduação o contato dos estudantes com situações clínicas simuladas, de alta complexidade ou urgências extremas, antes da inserção no ambiente de prática, pode diminuir seus sentimentos negativos, aperfeiçoar sua atuação, desenvolver a tomada de decisão e a empatia [8,10]. Nesse sentido, a simulação está se tornando cada vez mais um recurso predominante no ensino de enfermagem $[1,11]$.

No processo de aprendizado do estudante de enfermagem, a simulação promove desenvolvimento de competências essenciais à boa prática clínica como a segurança e a autoconfiança. A competência é resultado da combinação articulada entre conhecimento teórico (saber), a desenvoltura prática (agir) e a atitude (querer, poder, agir) que é influenciada pelo nível de segurança e autoconfiança apresentados pelo estudante em campo [12]. Na prática em campo, o estudante deve ter autoconfiança, se sentir capaz de realizar as atividades livre de erros, permitindo, dessa forma, que o professor e demais membros da equipe, acreditando em suas capacidades, o permitam partir para ação de modo mais autônomo [13].

O curto tempo dos estudantes em campo, a eventualidade e complexidade de algumas intervenções fazem com que a oportunidade de realizá-las seja reduzida. A simulação permite expor os estudantes a situações que geralmente não são vivenciadas nas práticas clínicas da graduação, embora sejam importantes para a atividade do profissional de enfermagem. Em uma estação simulada, os professores controlam as variáveis que influenciam uma situação e podem expor aos estudantes as mais diversas vivências e situações [12].

Além disso, esse recurso didático possibilita a partir da observação do comportamento dos estudantes diante dos seus pacientes e demais pessoas incluídas nos cenários simulados, o desenvolvimento de princípios relevantes a práxis da enfermagem relacionados aos direitos humanos e princípios fundamentais de bioética, tais como, justiça, autonomia, beneficência e não maleficência. Nessa perspectiva, a partir da simulação podem ser trabalhados aspectos que minimizem erros que poderiam causar danos em pacientes reais. Além disso, as reflexões dos estudantes e do professor que conduz o cenário auxiliam no aprendizado e no reforço ao cumprimento destes princípios [8].

As simulações de práticas clínicas também promovem no estudante maior capacidade de atuar, controlando fatores que poderiam afetar seu modo de atuação junto a um paciente real, fornecendo a ele subsídios para atuação segura e confiante [14,15]. 
Estudos comprovam que os estudantes que participaram de estações simuladas foram capazes de interagir melhor com as situações clínicas mais complexas e ganharam mais autoconfiança e conhecimento do que aqueles que possuíam somente o conteúdo teórico [16-18]. A simulação pode ser responsável por aumentar o nível de conhecimento prático dos estudantes, promovendo ganhos na autoconfiança, diminuindo o estresse e a tensão, além de possíveis erros nas posteriores intervenções com os pacientes [12,19-21].

Entre as intervenções que os enfermeiros atuam, a ressuscitação cardiopulmonar é uma das mais complexas e decisivas para sobrevivência do paciente. A rapidez, segurança e eficácia das intervenções dos profissionais de saúde podem garantir adequado retorno da ventilação e da circulação espontâneas, modificando a possibilidade de sobrevivência [22]. Para essa intervenção as equipes de saúde necessitam ser preparadas e ajustadas sendo que o enfermeiro é essencial nesse atendimento e deve estar preparado para iniciar o suporte básico de vida e auxiliar no avançado [22, 23].

Estima-se que ocorram no Brasil, cerca de 200.000 paradas cardiorrespiratórias por ano, metade delas em ambientes extra-hospitalares, informação que enfatiza a importância do preparo para o atendimento desses eventos fora de serviços hospitalares [23, 24].

Desse modo, entendendo a relevância da atuação do enfermeiro na ressuscitação cardiopulmonar extrahospitalar e compreendendo que a autoconfiança é uma variável de grande importância para a formação de enfermeiros $[19,20]$, elaborou-se a questão: "a simulação do atendimento à parada cardiorrespiratória em ambiente extra-hospitalar é efetiva para promover a autoconfiança de estudantes de enfermagem para atuar na ressuscitação cardiopulmonar?".

Assim é objetivo deste estudo: verificar a eficácia da simulação na autoconfiança de estudantes de enfermagem para atuação em ressuscitação cardiopulmonar extra-hospitalar.

\section{MÉTODOS}

Estudo quase experimental de grupo único, do tipo antes e depois de uma intervenção [25]. A simulação de atendimento à uma parada cardiorrespiratória extrahospitalar, em manequim, foi a intervenção avaliada nesta investigação. A variável dependente do estudo foi a autoconfiança dos estudantes de enfermagem para atuação nessa situação de emergência, medida pela Escala de Autoconfiança [26]. A hipótese desse tipo de estudo é que a melhora observada na variável dependente após intervenção (simulação) é resultado da própria experiência com a simulação.

Participaram da amostra do estudo estudantes do $2^{2}$ e $3^{\circ}$ anos do curso de Bacharelado em Enfermagem, com idade igual ou superior a 18 anos. O cenário do estudo foi uma universidade pública do interior fluminense.

Os critérios de exclusão foram: estudantes com formação técnica em enfermagem ou atuação profissional como técnico ou auxiliar de enfermagem; possuir treinamento de primeiros socorros ou participação ativa ou prévia em projetos de pesquisa ou extensão que tratam da temática emergência.

O cenário clínico simulado foi elaborado com base no roteiro proposto pelo Duke Human Simulation \& Patient Safety Center [27] e nas diretrizes de ressuscitação cardiopulmonar (RCP) em ambiente extra-hospitalar [26]. O cenário foi conduzido por um enfermeiro socorrista docente (facilitador) e dois estudantes do décimo período de graduação (atores), habilitados em suporte básico de vida. Foi utilizado o manequim de baixa fidelidade Mini Anne ${ }^{\circledR}$ Plus (Laerdal Corporation,Stockholm, Suécia) indicado para treinamento de pessoas leigas e estudantes. A duração da exposição do estudante ao cenário da simulação foi configurada em 20 minutos [28].

As ações esperadas no cenário foram estabelecidas de acordo com os passos da cadeia de sobrevivência relativos às ações de socorristas leigos [26]: reconhecer a parada cardiorrespiratória extra-hospitalar e acionar o serviço médico de emergência; iniciar ressuscitação cardiopulmonar imediata e de alta qualidade (compressões torácicas em ritmo de 100 a 120 por minuto, permitindo retorno do tórax após cada compressão de, no mínimo, cinco centímetros de profundidade); promover rápida desfibrilação; e atuar prontamente visando minimizar danos neurológicos. Em seguida foi submetido a teste piloto junto a quatro estudantes concluintes, para validação de conteúdo e aparência. Houve concordância de $100 \%$ entre os concluintes.

A coleta de dados foi realizada nos meses de abril e maio de 2016, utilizando os instrumentos: questionário para caracterização da amostra (incluiu idade, sexo, naturalidade e ano letivo em curso) e a Escala de Autoconfiança. Essa escala possui 12 itens, aplicados a três domínios (disfunção neurológica, disfunção respiratória e disfunção cardíaca), onde o respondente manifesta sua autoconfiança para reconhecer sinais e sintomas, avaliar com precisão o caso; intervir de modo apropriado e avaliar a eficácia das suas intervenções [29]. Para cada questão é solicitado uma das 
cinco possibilidades de resposta: nada confiante; pouco confiante; confiante; muito confiante e extremamente confiante. Para cada item do questionário é atribuído pontuações de 1 a 5 , sendo a menor pontuação atribuída à resposta nada confiante (1) e a maior, para extremamente confiante (5).

A dinâmica adotada para a coleta dos dados envolveu as seguintes etapas: inicialmente, antecedendo a simulação, foi preenchido o instrumento de identificação dos sujeitos. Na sequência, os estudantes participaram das atividades teóricas sobre a temática, conduzida pelo docente da equipe do cenário e a seguir foi aplicada a Escala de Autoconfiança. Após essa etapa, se iniciou a simulação com o cenário proposto, considerando o tempo de execução de até 20 minutos, incluindo a aplicação do debriefing estruturado [30], com questões norteadoras voltadas ao acionamento da cadeia de sobrevivência de ressuscitação cardiopulmonar extrahospitalar. Todos os estudantes participaram do cenário proposto individualmente. Após o término da simulação, a Escala de Autoconfiança foi novamente aplicada.

Os dados foram analisados utilizando o programa IBM SPSS Statistics 20, for Windows. As variáveis de caracterização demográfica foram apresentadas utilizando frequência absoluta e relativa. Para verificar a evolução dos níveis de autoconfiança dos estudantes para a atuação em parada cardiorrespiratória extrahospitalar foi utilizado o teste de Homogeneidade Marginal. Para comparar a diferença entre as médias nos domínios da Escala de Autoconfiança, antes e depois da simulação, foi aplicado o teste de postos sinalizados de Wilcoxon. O teste de confiabilidade Alfa de Cronbach foi usado para a Escala de Autoconfiança antes e depois. O nível de significância adotado para os testes estatísticos foi de $5 \%(p<0,05)$.

O estudo foi aprovado por Comitê de Ética em Pesquisa, conforme diretrizes que regulamentam as pesquisas envolvendo seres humanos, constantes na Resolução no 466 de 12 de dezembro de 2012, recebendo parecer favorável $\mathrm{n}$ o 1.410 .658 . Todos os participantes foram informados sobre a pesquisa e assinaram as duas vias do Termo de Consentimento Livre e Esclarecido. O estudo foi registrado e aprovado no Registro Brasileiro de Ensaios Clínicos. Registro Brasileiro de Ensaio Clínico: RBR-25gnjc.

\section{RESULTADOS}

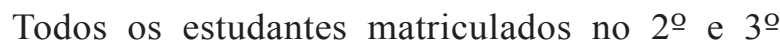
anos do Curso de Bacharelado em Enfermagem foram convidados a participar do estudo. Entre os 83 estudantes matriculados no período, 33 $(39,7 \%)$ manifestaram interesse em participar desta investigação, um estudante foi excluído por possuir formação técnica em Enfermagem, totalizando 32 sujeitos na casuística (Figura 1). O tempo médio de preenchimento dos instrumentos foi 15 minutos.

Dos 32 estudantes de Enfermagem incluídos no estudo, $28(87,5 \%)$ eram do sexo feminino. A faixa etária variou de 18 a 38 anos (média de idade 23,3 $\pm 5,1$ anos). Com relação à naturalidade, houve predomínio do estado do Rio de Janeiro (59,4\%), embora tenha sido observada diversidade de estados de nascimento dos estudantes: São Paulo, 12,7\%; Minas Gerais e Pará, 9,3\%; Alagoas, Bahia e Rio Grande do Sul, $3,1 \%$. Quanto ao ano que cursavam Bacharelado em

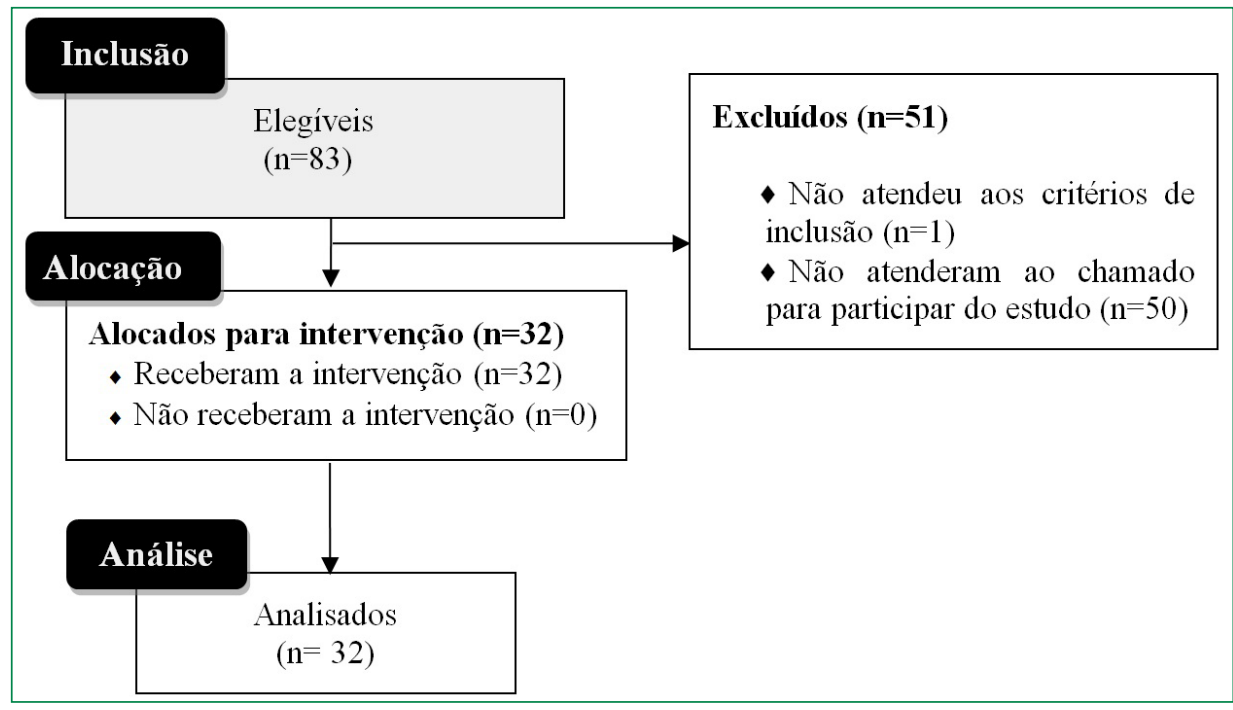

Figura 1. Diagrama representativo do fluxo de participantes nas etapas do estudo conforme enunciado CONSORT [31]. 
Enfermagem, os estudantes se distribuíram igualmente entre segundo e terceiro ano.

Antes da simulação, a maioria dos estudantes indicou confiança para reconhecer sinais e sintomas de um evento cardíaco $(68,8 \%)$ e respiratório $(53,1 \%)$. Já para o reconhecimento de sinais e sintomas de um evento neurológico, a maioria relatou estar pouco autoconfiante $(46,9 \%)$.

Quanto à autoconfiança para avaliar com precisão um indivíduo, os estudantes relataram estar pouco confiantes para uma situação cardiológica $(43,8 \%)$ e neurológica $(50,0 \%)$. Em relação à disfunção respiratória, $53,1 \%$ dos estudantes relataram estar confiantes na avaliação.

A maioria dos estudantes demonstrou estar pouco confiante para atuação numa disfunção cardíaca $(53,1 \%)$, respiratória $(50,0 \%)$ e neurológica $(56,3 \%)$. A confiança em avaliar a eficácia das intervenções realizadas também predominou entre os participantes deste estudo: $65,6 \%$ para as cardíacas; $59,4 \%$ para as respiratórias e $53,1 \%$ para as neurológicas.

Após o uso de estratégias de simulação, os estudantes informaram estar confiantes para reconhecer sinais e sintomas de um evento cardíaco: muito confiantes $(50,0 \%)$, confiantes $(25,0 \%)$ e extremamente confiantes $(25,0 \%) ; 46,9 \%$ relataram estar muito confiantes para reconhecer sinais e sintomas de um evento respiratório e 50,0\% relataram estar confiantes para reconhecer sinais e sintomas de um evento neurológico. Quanto à capacidade de avaliar com precisão um evento cardíaco, 50,0\% afirmaram estar confiantes e $43,8 \%$ muito autoconfiantes na avaliação de eventos respiratórios e neurológicos.

Cerca de $40,0 \%$ dos estudantes estavam muito confiantes para realizar intervenções cardíacas e neurológicas após a simulação e 46,9\% estavam confiantes para intervir em situação respiratória. Em relação a avaliar a eficácia das intervenções, os estudantes afirmaram ter confiança para avaliação dos resultados de intervenções cardíacas $(43,8 \%)$, respiratórias $(40,6 \%)$ e neurológicas $(43,8 \%)$. O teste de homogeneidade marginal revelou diferenças estatisticamente significantes $(\mathrm{p}<0,001)$ nas respostas de todas as questões da Escala de Autoconfiança, quando comparadas antes e depois da simulação (Tabela 1).

Tabela 1. Percentual dos estudantes segundo resposta na Escala de Autoconfiança, antes e depois da simulação ( $\mathrm{n}=32$ ).

\begin{tabular}{|c|c|c|c|c|c|c|c|}
\hline \multirow{2}{*}{ Questões } & \multicolumn{6}{|c|}{ \% de estudantes segundo níveis de autoconfiança } & \multirow{2}{*}{ p "valor } \\
\hline & & $\mathrm{NC}^{*}$ & $\mathrm{PC}^{+}$ & $\mathrm{C}^{\ddagger}$ & MC§ & ECII & \\
\hline \multirow{2}{*}{$\begin{array}{l}\text { Q1. Quão confiante está de ser capaz de reconhecer sinais e sintomas de um evento } \\
\text { cardíaco? }\end{array}$} & Antes & 3,1 & 21,9 & 68,8 & 3,1 & 3,1 & \multirow{2}{*}{$<0,001$} \\
\hline & Depois & - & - & 25,0 & 50,0 & 25,0 & \\
\hline \multirow{2}{*}{$\begin{array}{l}\text { Q2. Quão confiante está de ser capaz de reconhecer sinais e sintomas de um evento } \\
\text { respiratório? }\end{array}$} & Antes & 9,4 & 31,3 & 53,1 & 6,2 & - & \multirow{2}{*}{$<0,001$} \\
\hline & Depois & - & 3,1 & 37,5 & 46,9 & 12,5 & \\
\hline \multirow{2}{*}{$\begin{array}{l}\text { Q3. Quão confiante está de ser capaz de reconhecer sinais e sintomas de um evento } \\
\text { neurológico? }\end{array}$} & Antes & 21,9 & 46,9 & 28,1 & 3,1 & - & \multirow{2}{*}{$<0,001$} \\
\hline & Depois & - & 9,4 & 50,0 & 34,4 & 6,2 & \\
\hline \multirow{2}{*}{$\begin{array}{l}\text { Q4. Quão confiante está de ser capaz de avaliar com precisão um indivíduo com dor } \\
\text { torácica? }\end{array}$} & Antes & 15,6 & 43,8 & 34,4 & 6,2 & - & \multirow{2}{*}{$<0,001$} \\
\hline & Depois & - & 6,2 & 50,0 & 34,4 & 9,4 & \\
\hline \multirow{2}{*}{$\begin{array}{l}\text { Q5. Quão confiante está de ser capaz de avaliar com precisão um indivíduo com dor } \\
\text { dispneia? }\end{array}$} & Antes & - & 34,4 & 53,1 & 9,4 & 3,1 & \multirow{2}{*}{$<0,001$} \\
\hline & Depois & - & 3,1 & 34,4 & 43,8 & 18,7 & \\
\hline \multirow{2}{*}{$\begin{array}{l}\text { Q6. Quão confiante está de ser capaz de avaliar com precisão um indivíduo com } \\
\text { alteração do estado mental? }\end{array}$} & Antes & 6,3 & 50,0 & 34,4 & 9,3 & - & \multirow{2}{*}{$<0,001$} \\
\hline & Depois & - & 9,3 & 43,8 & 31,3 & 15,6 & \\
\hline \multirow{2}{*}{$\begin{array}{l}\text { Q7. Quão confiante está de ser capaz de intervir apropriadamente num indivíduo com } \\
\text { dor torácica? }\end{array}$} & Antes & 25,0 & 53,1 & 18,8 & 3,1 & - & \multirow{2}{*}{$<0,001$} \\
\hline & Depois & - & 12,5 & 40,6 & 34.4 & 12,5 & \\
\hline \multirow{2}{*}{$\begin{array}{l}\text { Q8. Quão confiante está de ser capaz de intervir apropriadamente num indivíduo com } \\
\text { dispneia? }\end{array}$} & Antes & 9,4 & 50,0 & 31,3 & 6,2 & 3,1 & \multirow{2}{*}{$<0,001$} \\
\hline & Depois & - & 6,3 & 28,1 & 46,9 & 18,7 & \\
\hline \multirow{2}{*}{$\begin{array}{l}\text { Q9. Quão confiante está de ser capaz de intervir apropriadamente num indivíduo com } \\
\text { alteração do estado mental? }\end{array}$} & Antes & 18,8 & 56,3 & 18,7 & 6,3 & - & \multirow{2}{*}{$<0,001$} \\
\hline & Depois & - & 12,5 & 40,6 & 31,3 & 15,6 & \\
\hline \multirow{2}{*}{$\begin{array}{l}\text { Q10. Quão confiante está de ser capaz de avaliar a eficácia das suas intervenções num } \\
\text { indivíduo com dor torácica? }\end{array}$} & Antes & 18,8 & 65,5 & 9,4 & 6,3 & - & \multirow{2}{*}{$<0,001$} \\
\hline & Depois & - & 9,4 & 43,7 & 37,5 & 9,4 & \\
\hline \multirow{2}{*}{$\begin{array}{l}\text { Q11. Quão confiante está de ser capaz de avaliar a eficácia das suas intervenções num } \\
\text { indivíduo com dispneia? }\end{array}$} & Antes & 15,6 & 59,4 & 15,6 & 9,4 & - & \multirow{2}{*}{$<0,001$} \\
\hline & Depois & - & 6,3 & 40,6 & 43,8 & 9,3 & \\
\hline \multirow{2}{*}{$\begin{array}{l}\text { Q12. Quão confiante está de ser capaz de avaliar a eficácia de suas intervenções num } \\
\text { indivíduo com alteração do estado mental? }\end{array}$} & Antes & - & 25,0 & 53,1 & 15,6 & 6,3 & \multirow{2}{*}{$<0,001$} \\
\hline & Depois & 3,1 & 9,4 & 43,8 & 40,6 & 3,1 & \\
\hline
\end{tabular}

${ }^{*} \mathrm{NC}=$ nada confiante; ${ }^{+\mathrm{PC}}=$ pouco confiante; ${ }^{\ddagger} \mathrm{C}=$ confiante; $\$ \mathrm{MC}=$ muito confiante; $\| \mathrm{EC}=$ extremamente confiante; ${ }^{~} \mathrm{valor}$ de $\mathrm{p}$ obtido através do teste de homogeneidade marginal 
Com relação à Escala de Autoconfiança, na amostra pesquisada foram obtidos altos valores de confiabilidade do instrumento antes e depois da intervenção (Alfa de Cronbach 0,89 e 0,93, respectivamente), indicando ótima consistência interna.

A Figura 2 mostra que os níveis de menor confiança predominaram antes da simulação. Para todas as questões analisadas, após a execução do cenário clínico simulado, os níveis de maior confiança foram observados, com destacado aumento do percentual de estudantes com maiores pontuações nas respostas.

$\mathrm{Na}$ comparação dos domínios disfunção neurológica, disfunção cardíaca e disfunção respiratória, houve diferença estatística significativa nas médias antes e depois da realização da simulação (Tabela 2).

\section{DISCUSSÃO}

O estudo mostrou que a simulação foi efetiva na promoção da autoconfiança dos estudantes de enfermagem para atuação emergencial em parada cardiorrespiratória extra hospitalar. Houve aumento da pontuação da Escala de Autoconfiança em todos domínios fisiológicos (disfunção cardíaca, disfunção respiratória e disfunção neurológica) depois da atividade. Esses resultados corroboram as indicações de que a simulação é uma estratégia educativa muito útil, pois permite desenvolver atributos importantes para a prática clínica como a autoconfiança, a empatia, o desenvolvimento da liderança $[8,13]$. Tais atributos auxiliam na tomada de decisões em situações com alto grau de estresse e complexidade [32-34].

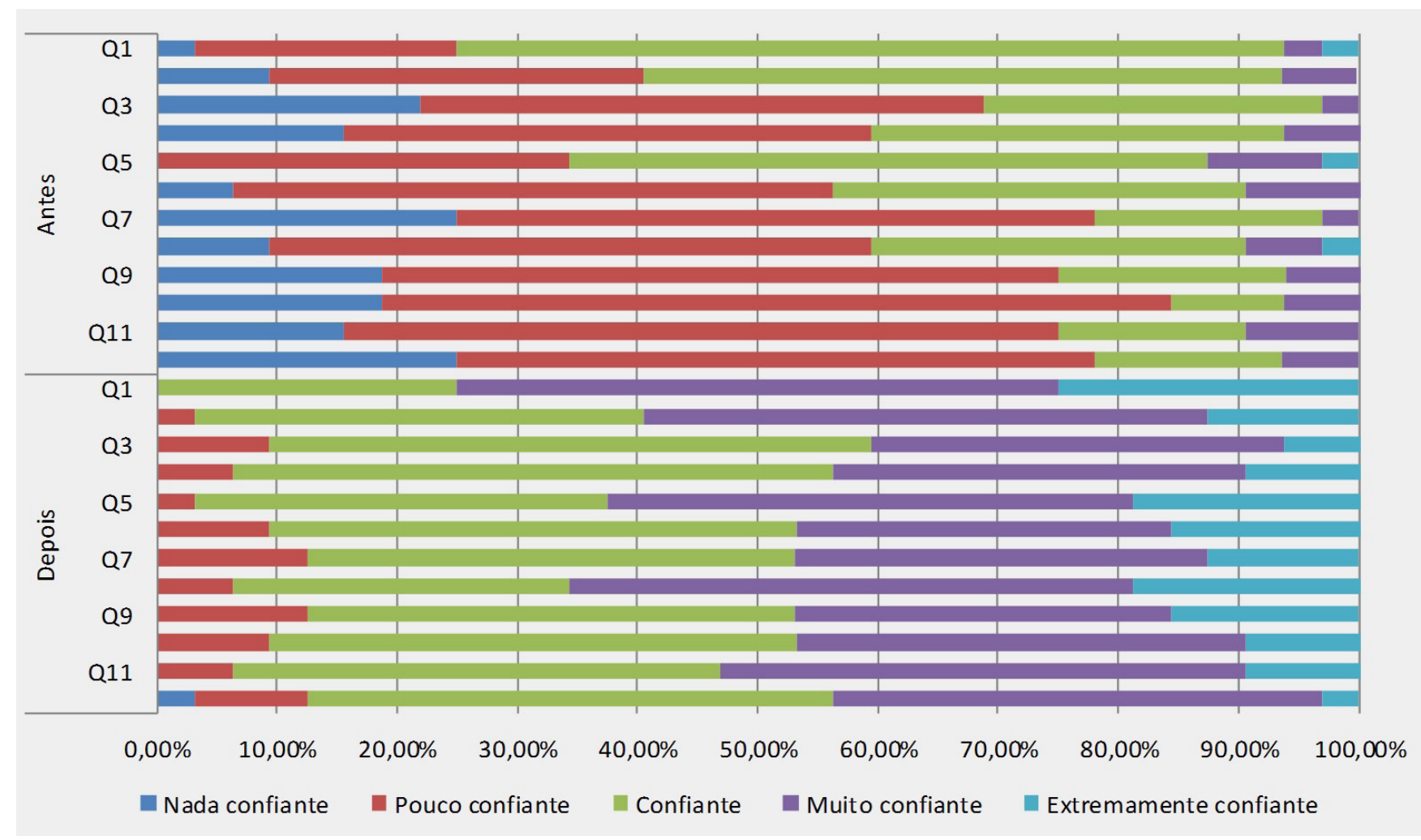

Figura 2. Distribuição das respostas dos estudantes na Escala de Autoconfiança antes e depois da simulação.

Tabela 2. Avaliação da autoconfiança por domínios fisiológicos antes e depois da simulação $(n=32)$.

\begin{tabular}{lcccccc}
\hline & \multicolumn{2}{c}{ Antes } & \multicolumn{3}{c}{ Depois } & $\mathbf{p}^{\mathbf{a}}$ \\
\cline { 2 - 3 } & Média & DP & Média & DP & $<0,001$ \\
Disfunção neurológica & 9,81 & 2,18 & 14,53 & 2,29 & $<0,001$ \\
Disfunção respiratória & 9,72 & 2,26 & 14,56 & 2,82 & $<0,001$ \\
\hline Disfunção cardíaca & 8,38 & 2,59 & 13,84 & 2,84 & \\
\hline
\end{tabular}

a Teste de postos sinalizados de Wilcoxon. 
É essencial o desenvolvimento de habilidades crítico-reflexivas de natureza clínica concomitantemente a alta velocidade de resposta a uma situação de emergência. A simulação surge como importante ferramenta para prover essas habilidades aos estudantes de enfermagem [35,36]. Nesse sentido, a Organização Mundial da Saúde, recomenda que as instituições de ensino que formam profissionais de saúde devem adotar a simulação na educação e treino de seus estudantes [37].

Estudo desenvolvido com estudantes chineses do terceiro ano do curso de graduação em enfermagem revelou que a simulação auxiliou na diminuição do tempo de resposta para o início das intervenções nos contextos da emergência e da terapia intensiva [17]. Em outros dois estudos, os estudantes relataram perceber melhorias nas habilidades de comunicação aplicadas à prática clínica, refletindo em melhores resultados para os pacientes $[18,37,38]$.

Em outro estudo, realizado com 32 estudantes de enfermagem buscando avaliar sua percepção sobre a simulação como estratégia educativa, foi revelado que os estudantes percebiam a simulação como um método mais eficaz para ensinar habilidades clínicas do que a abordagem didática tradicional, concluindo que este é um método satisfatório para o processo de formação [39].

No geral, a simulação é uma estratégia muito bem aceita pelos estudantes, pois permite construir um rico espaço para reflexões das práticas profissionais, incrementando o diálogo teórico-prático em cenários e situações-problemas pertinentes ao ciclo de conhecimentos do estudante $[13,40,41]$.

Investigação desenvolvida com 52 estudantes de enfermagem, que foram expostos a atividades teóricas e simulações para correta intervenção em situações de emergência constatou que a autoconfiança global na Escala de Autoconfiança aumentou de 2,35 pontos para 3,34 pontos após as simulações. O estudo concluiu que a simulação é uma boa estratégia para o desenvolvimento de confiança para intervir em uma situação de emergência [42].

$\mathrm{Na}$ atual investigação observou-se maior aumento no valor médio dos domínios da Escala de Autoconfiança: 4,72 para disfunção neurológica, 4,84 para a respiratória e 5,46 para a cardíaca (Tabela 2). Em estudo realizado com estudantes de medicina, também foi verificado que a simulação promoveu uma significativa melhora na confiança para lidar com situações de emergência [19].

A autoconfiança é uma atitude relacionada às experiências repetidas e os indivíduos com níveis mais elevados de autoconfiança têm maior probabilidade de executar intervenções bem-sucedidas [4,31]. Assim, o desenvolvimento da autoconfiança de graduandos em enfermagem é de extrema importância para que haja maior segurança nas suas tomadas de decisões clínicas futuras, que impactam na probabilidade de sobrevida do paciente $[31,41]$.

Nesse sentido, o aumento na autoconfiança dos indivíduos que participaram deste estudo indica que a simulação é alternativa promissora para promover uma experiência prática no manejo clínico correto das emergências, sem colocar em risco a integridade física dos pacientes. Portanto, o uso de simulações clínicas de emergência para desenvolver habilidades de raciocínio clínico de estudantes é uma importante e promissora ferramenta com impactos positivos que vão além da confiança adquirida em seu processo $[4,43]$.

Em um estudo realizado nos Estados Unidos com 90 estudantes de enfermagem, com objetivo de descrever o uso de uma estratégia educacional envolvendo simulação para preparar estudantes de enfermagem a lidar com situações de emergência, foi observado que $95 \%$ dos estudantes recomendaram a simulação como estratégia educativa, pois concordam que a simulação ajuda a aumentar o conhecimento e a confiança em lidar com uma emergência clínica [44]. Outra vantagem apontada pelos estudantes é que ela permite que os estudantes possam praticar os conhecimentos teóricos adquiridos com feedback instantâneo, tudo isso em um ambiente controlado sem promover riscos ao paciente $[14,45,46]$. Sobre o feedback e debriefing após os cenários de simulação, estudo aponta que as emoções devem consideradas, principalmente por causa do impacto emocional na aprendizagem e no desempenho do estudante [33].

Para a prática de enfermagem o estudo fornece evidências acerca do uso da simulação como ferramenta pedagógica para a formação de enfermeiros, contribuindo com o ganho na autoconfiança e maior segurança para atuação em contextos de complexidade extrema, como o de parada cardiorrespiratória.

Para a saúde, os achados deste estudo reforçam a necessidade de treinamento de habilidades técnicas associadas ao desenvolvimento de competências emocionais que podem ser desenvolvidas em cenários simulados aplicados na graduação.

A limitação do estudo está no tamanho da amostra, embora os resultados tenham ratificado os achados de outras pesquisas realizadas em contexto nacional e internacional. O tempo de manutenção da autoconfiança 
após a participação no cenário simulado ainda é pouco explorado. Evidencia-se, portanto, a necessidade de realização de novos estudos, com avaliação da autoconfiança em períodos diversificados após a exposição à simulação para uma maior abrangência dos resultados.

Neste estudo, a simulação do atendimento a uma parada cardiorrespiratória extra-hospitalar promoveu ganhos na autoconfiança dos estudantes de enfermagem para atuação em emergência, sustentando a hipótese desta investigação, que propõe que a simulação aumenta a autoconfiança de estudantes de enfermagem para atuar na ressuscitação cardiopulmonar.

\section{NOTAS}

Declaração de conflito de interesses

Os autores declaram não haver conflitos de interesses relevantes ao conteúdo deste estudo.

Contribuições dos autores

Todos os autores fizeram contribuições substanciais para concepção, ou delineamento, ou aquisição, ou análise ou interpretação de dados; e redação do trabalho ou revisão crítica; e aprovação final da versão para publicação.

Disponibilidade dos dados e responsabilidade pelos resultados

Todos os autores declaram ter tido total acesso aos dados obtidos e assumem completa responsabilidade pela integridade destes resultados.

\section{REFERÊNCIAS}

1. Martins JCA, Mazzo A, Baptista RCN, Coutinho VRD, Godoy S, Mendes IAC, Trevizan MA. A experiência clínica simulada no ensino de enfermagem: retrospectiva histórica. Acta Paul Enferm. 2012;25(4):619-25. https://doi. org/10.1590/s0103-21002012000400022

2. Alamrani MH, Alammar KA, Alqahtani SS, Salem OA. Comparing the effects of simulation-based and traditional teaching methods on the critical thinking abilities and self-confidence of nursing students. J Nurs Res. 2018;26(3):152-7. https://doi.org/10.1097/jnr.0000000000000231

3. Perry, P. Concept analysis: confidence/self-confidence. Nurs Forum. 2011;46(4):218-30. https://doi.org/10.1111/ j.1744-6198.2011.00230.x

4. Menezes SSC, Corrêa CG, Silva RCG, Cruz DAML. Raciocínio clínico no ensino de graduação em enfermagem: revisão de escopo. Rev Esc Enferm USP. 2015;49(6):1037-44. https://doi.org/10.1590/s0080-62342010000300019

5. Brandão CFS, Cecilio-Fernandes D. Importância e desafios do treinamento simulado em saúde. Sci Med. 2018;28(1):ID30102. https://doi.org/10.15448/1980-6108.2018.1.3010

6. Perbone JG, Carvalho EC. Sentimentos do estudante de enfermagem em seu primeiro contato com pacientes. Rev Bras Enferm. 2011;64(2):343-7. https://doi.org/10.1590/s0034-71672011000200019

7. Kiernan LC. Evaluating competence and confidence using simulation technology. Nursing. 2018;48(10):45-52. https://doi.org/10.1097/01.nurse.0000545022.36908.f3

8. Costa RRO, Medeiros SM, Martins JCA, Coutinho VRD. A simulação no ensino de enfermagem: reflexões e justificativas a luz da bioética e dos direitos humanos. Acta Bioeth. 2018;24(1):31-8. https://doi.org/10.4067/s1726$569 \times 2018000100031$

9. Costa RRO, Medeiros SM, Martins JCA, Enders BC, Lira ALBC, Araújo MS. A simulação no ensino de enfermagem: uma análise conceitual. Rev Enferm Centro-Oeste Mineiro. 2018;8:e1928. https://doi.org/10.19175/recom.v8i0.1928

10. Oliveira SN, Prado ML, Kempfer SS, Waterkemper R, Morera JAC, Bernardi MC. A pedagogia por trás da experiência clínica simulada: uma percepção de estudantes de enfermagem. Rev Iberoam Educ Investi Enferm. 2015;5(3):56-3.

11. Bias CGS, Agostinho LS, Coutinho RP, Barbosa GS. Simulation in emergency nursing education: an integrative review. J Nurs Educ Pract. 2016;6(12):12-7.

12. Moura ECC, Caliri MHL. Simulação para desenvolvimento da competência clínica de avaliação de risco para úlcera por pressão. Acta Paul Enferm. 2013;26(4):369-75. https://doi.org/10.1590/s0103-21002013000400011

13. Martins JCA. Learning and development in simulated practice environments. Rev Enf Ref. 2017;serIV(12):155-62.

14. Lestander Ö, Letho N, Engström Å. Nursing students' perceptions of learning after high fidelity simulation: effects of a three-step post-simulation reflection model. Nurse Educ Today. 2015;40:219-24. https://doi.org/10.1016/j. nedt.2016.03.011 
15. Teixeira CRS, Kusumota L, Pereira MCA, Braga FTMM, Gaioso VP, Zamarioli CM, Carvalho EC. Anxiety and performance of nursing students in regard to assessment via clinical simulations in the classroom versus filmed assessments. Invest Educ Enferm. 2014;32(2):270-9. https://doi.org/10.17533/udea.iee.v32n2a10

16. Shinnick MA, Woo MA. Does nursing student self-efficacy correlate with knowledge when using human patient simulation? Clin Simul Nurs. 2014;10(2):e71-9. https://doi.org/10.1016/j.ecns.2013.07.006

17. Chen J, Yang J, Hu F, Yu SH, Yang BX, Liu Q, Zhu XP. Standardised simulation-based emergency and intensive care nursing curriculum to improve nursing students' performance during simulated resuscitation: a quasi-experimental study. Intensive Crit Care Nurs. 2018;46:51-6. https://doi.org/10.1016/j.iccn.2018.02.003

18. Tobase L, Peres HHC, Gianotto-Oliveira R, Smith N, Polastri TF, Timerman S. The effects of an online basic life support course on undergraduate nursing students' learning. Int J Med Educ. 2017;8:309-13. https://doi.org/10.5116/ ijme.5985.cbce

19. Muniandy RK, Nyein KK, Felly M. Improving the self-confidence level of medical undergraduates during emergencies using high fidelity simulation. Med J Malaysia. 2015;70(5):300-2.

20. Gharaibeh B, Hweidi I, Al-Smadi A. Attitudes and perception of baccalaureate nursing students toward educational simulation. Cogent Education. 2017;4(1):1360063. https://doi.org/10.1080/2331186x.2017.1360063

21. Martínez-Castillo F, Matus-Miranda R. Desarrollo de habilidades con simulación clínica de alta fidelidad. Perspectiva de los estudiantes de enfermería. Enferm Univ. 2015;12(2):93-8. https://doi.org/10.1016/j.reu.2015.04.003

22. Almeida AO, Araujo IEM, Dalri MCB, Araujo S. Conhecimento teórico dos enfermeiros sobre parada e ressuscitação cardiopulmonar, em unidades não hospitalares de atendimento à urgência e emergência. Rev Latinoam Enferm. 2011;19(2):261-8. https://doi.org/10.11606/d.7.2008.tde-16042008-144419

23. Nogueira LS, Wilson AMMM, Karakhanian ACM, Parreira EV, Machado VMP, Mira VL. Avaliação dos conhecimentos e habilidades em ressuscitação cardiopulmonar assimilados por profissionais da atenção primária em saúde. Sci Med. 2018;28(1):ID28843. https://doi.org/10.15448/1980-6108.2018.1.28843

24. Gonzalez MM, Timerman S, Gianotto-Oliveira R, Polastri TF, Canesin MF, Schimidt A, Siqueira AW, Pispico A, Longo A, Pieri A, Reis A, Tanaka ACS, Santos AM, Quilici AP, Ribeiro ACL, Barreto ACP, Pazin-Filho A, Timerman A, Machado CA, Franchin Neto C, Miranda CH, Medeiros CR, Malaque CMS, Bernoche C, Gonçalves DM, Sant'Ana DG, Osawa EA, Peixoto E, Arfelli E, Evaristo EF, Azeka E, Gomes EP, Wen FH, Ferreira FG, Lima FG, Mattos FR, Galas FG, Marques FRB, Tarasoutchi F, Mancuso FJN, Freitas GR, Feitosa-Filho GS, Barbosa GC, Giovanini GR, Miotto HC, Guimarães HP, Andrade JP, Oliveira-Filho J, Fernandes JG, Moraes Junior JBMX, Carvalho JJF, Ramires JAF, Cavalini JF, Teles JMM, Lopes JL, Lopes LNGD, Piegas LS, Hajjar LA, Brunório L, Dallan LAP, Cardoso LF, Rabelo MMN, Almeida MFB, Souza MFS, Favarato MH, Pavão MLRC, Shimoda MS, Oliveira Junior MT, Miura N, Filgueiras Filho NM, Pontes-Neto OM, Pinheiro PAPC, Farsky OS, Lopes RD, Silva RCG, Kalil Filho R, Gonçalves RM, Gagliardi RJ, Guinsburg R, Lisak S, Araújo S, Martins SCO, Lage SG, Franchi SM, Shimoda T, Accorsi TD, Barral TCN, Machado TAO, Scudeler TL, Lima VC, Guimarães VA, Sallai VS, Xavier WS, Nazima W, Sako YK. I Diretriz de Ressuscitação Cardiopulmonar e Cuidados Cardiovasculares de Emergência da Sociedade Brasileira de Cardiologia. Arq. Bras. Cardiol., Arq. Bras. Cardiol. 2013;101(2 supl. 3):1-221. https://doi.org/10.5935/abc.2013s006

25. Gray J, Grove SK, Sutherland S. Selecting a quantitative research design. In: Gray JR, Grove SK, Sutherland S. Burns and Grove's the practice of nursing research: appraisal, synthesis, and generation of evidence. 8th ed. Pennsylvania: Saunders; 2016. p. 214-63.

26. Neumar RW, Shuster M, Callaway CW, Gent LM, Atkins DL, Bhanji F, Brooks SC, de Caen AR, Donnino MW, Ferrer JM, Kleinman ME, Kronick SL, Lavonas EJ, Link MS, Mancini ME, Morrison LJ, O'Connor RE, Samson RA, Schexnayder SM, Singletary EM, Sinz EH, Travers AH, Wyckoff MH, Hazinski MF. Part 1: Executive summary: 2015 American Heart Association Guidelines update for cardiopulmonary resuscitation and emergency cardiovascular care. Circulation. 2015;132(18 Suppl 2):S315-67. https://doi.org/10.1161/cir.0000000000000252

27. Duke Human Simulation and Patient Safety Center. Duke University template for case-based scenarios [Internet]. Durham: Duke University and Duke University Health System, 2018 [capturado em 11 abr. 2019]. Disponível em: https://anesthesiology.duke.edu/?page_id=825706. https://doi.org/10.22462/5.6.2017.1

28. Maia ER, Gonçalves Júnior J, Lima EP, Campos W, Jovino EM, Fernandes FF, Lacerda VRC. Conhecimentos em atenção pré-hospitalar e suporte básico de vida por estudantes recém-ingressos de medicina. Rev Bras Educ Med. 2014;38(1):59-64. https://doi.org/10.1590/s0100-55022014000100008

29. Martins JCA, Baptista RCN, Coutinho VRD, Mazzo A, Rodrigues MA, Mendes IAC. Autoconfiança para intervenção em emergências: adaptação e validação cultural da Self-confidence Scale em estudantes de Enfermagem. Rev Latinoam Enfermagem. 2014;22(4):554-61. https://doi.org/10.1590/0104-1169.3128.2451 
30. Palaganas JC, Fey M, Simon R. Structured debriefing in simulation-based education. AACN Adv Crit Care. 2016;27(1):78-85. https://doi.org/10.4037/aacnacc2016328

31. Moher D, Hopewell S, Schulz KF, Montori V, Gøtzsche PC, Devereaux PJ, Elbourne D, Egger M, Altman DG. CONSORT 2010 Explanation and Elaboration: updated guidelines for reporting parallel group randomised trials. BMJ. 2010;340:c869. https://doi.org/10.1136/bmj.c869

32. Martins J, Mazzo A, Mendes I, Rodrigues M. A simulação no ensino de enfermagem. Coimbra: Enfermagem/Escola Superior de Enfermagem de Coimbra; 2014.

33. Baptista RCN, Martins JCA, Pereira MFCR, Mazzo A. Simulação de alta-fidelidade no Curso de Enfermagem: ganhos percebidos pelos estudantes. Rev Enf Ref. 2014;IV(1):135-44. https://doi.org/10.12707/riii13169

34. Carvalho-Filho MA, Schaafsma ES, Tio RA. Debriefing as an opportunity to develop emotional competence in health profession students: faculty, be prepared! Sci Med. 2018;28(1):ID28805. http://doi.org/10.15448/19806108.2018.1.28805

35. Kowitlawakul Y, Chow YL, Salam ZH, Ignacio J. Exploring the use of standardized patients for simulation-based learning in preparing advanced practice nurses. Nurs Educ Today. 2015;35(7):894-9. https://doi.org/10.1016/j. nedt.2015.03.004

36. Aqel AA, Ahmad MM. High-fidelity simulation effects on CPR knowledge, skills, acquisition, and retention in nursing students. Worldviews Evid Based Nurs. 2014;11(6):394-400. https://doi.org/10.1111/wvn.12063

37. World Health Organization. Transforming and scaling up health professionals' education and training: WHO education guidelines 2013. Geneva: WHO; 2013.

38. Karlsen MW, Gabrielsen AK, Falch AL, Stubberud DG. Intensive care nursing students' perceptions of simulation for learning confirming communication skills: a descriptive qualitative study. Intensive Crit Care Nurs. 2017;42:97-104. https://doi.org/10.1016/j.iccn.2017.04.005

39. Baker VO, Sturdivant S, Masters C, McCarthy M, Carlson J. Undergraduate cardiac arrest team training. Clin Teach. 2015;12(4):255-9. https://doi.org/10.1111/tct.12303

40. Dickinson T, Hopton J, Piling M. An evaluation of nursing students' perceptions on the efficacy of high fidelity clinical simulation to enhance their confidence, understanding and competence in managing psychiatric emergencies. J Clin Nurs. 2016;25(9-10):1476-8. https://doi.org/10.1111/jocn.13211

41. Teixeira CRS, Pereira MCA, Kusumota L, Gaioso VP, Mello CL, Carvalho EC. Evaluation of nursing students about learning with clinical simulation. Rev Bras Enferm. 2015;68(2):311-9.

42. Carvalho DPSRP, Azevedo IC, Cruz GKP, Mafra GAC, Rego ALC, Vitor AF, Santos VEP, Cogo ALP, Ferreira Júnior MA. Strategies used for the promotion of critical thinking in nursing undergraduate education: a systematic review. Nurse Educ Today. 2017;57:103-7. https://doi.org/10.1016/j.nedt.2017.07.010

43. Martins JCA, Baptista RCN, Coutinho VRD, Carvalho E, Rosebal Y, Correia NC, Mazzo A, Rodrigues MA, Mendes IAC. Theoretical and simulation classes in the emergency nursing curriculum in Cape Verde: effect on the self-confidence to intervene in emergencies. J Nurs Educ Pract. 2014;4(8):26-33. https://doi.org/10.5430/jnep. v4n 8 p26

44. Shin S, Park JH, Kim JH. Effectiveness of patient simulation in nursing education: meta-analysis. Nurse Educ Today. 2015;35(1):176-82. https://doi.org/10.1016/j.nedt.2014.09.009

45. Kaplan BG, Connor A, Ferranti EP, Holmes L, Spencer L. Use of an emergency preparedness disaster simulation with undergraduate nursing students. Public Health Nurs. 2011;29(1):44-51. https://doi.org/10.1111/j.15251446.2011.00960.x

46. Tivener KA, Gloe DS. The effect of high-fidelity Cardiopulmonary Resuscitation (CPR) simulation on athletic training student knowledge, confidence, emotions, and experiences. Athl Train Educ J. 2015;10(2):103-12. https://doi. org/10.4085/1002103 C 